\title{
Dynamics of Complex Mechanical Systems Based on Lie Groups and Lie algebra
}

\author{
Shao Bing ${ }^{1, a}$, Yuan Entao ${ }^{1}$ \\ ${ }^{1}$ School of Mechanical, Shanghai Dianji University, Shanghai 201306, China
}

\begin{abstract}
Adjoint transformations and adjoint operators of Lie groups and Lie algebra are discussed in this paper to study the recursive dynamics of complex systems. The dynamics based on Lie groups and Lie algebra of tree topology systems is then developed. Efficient dynamics algorithms for closed-loop systems and flexible systems can be developed. Simulation results show that the dynamic problems can be solved quickly and efficiently using the method. This study provides a foundation for real-time control based on dynamics.
\end{abstract}

\section{Introduction}

With the development of modern science and technology, there has been an engineering object composed by a large number of objects, such as vehicles, robots, aerospace vehicles. These complex mechanical systems contact with each other in a variety of manners. Multi-body system dynamics is the large-scale complex systems researching the forward, backward and mixed problem. Inverse dynamics can be applied to optimize the mechanical design and control [1]. By calculating the inverse kinematics, the supporting force and moments can be obtained. This complete information used in mechanical design can be more accurate than the traditional static design method. It can optimize the design and reduce the quality of the system and energy consumption. At the same time, the multi-body system inverse dynamics are applied to control. Forward dynamics in modern engineering technology is mainly used for virtual simulation which can save huge costs of design, manufacture and test.

The rapid development of large-scale systems based on the dynamics and efficiency of real-time control requires a substantial increase in dynamics [2]. Based on the need of high efficiency, recursive dynamical algorithms are obtained. Computational dynamics recursive algorithm is $\mathrm{O}(\mathrm{N})$, where $\mathrm{N}$ is the degrees of freedom, namely freedom increases linearly with the amount of calculation. For the inverse dynamics problem, first developed in the $\mathrm{O}(\mathrm{N})$ algorithm is Newton-Euler (referred to as NE) formula [3]. Rodriguez Guillermo implemented $\mathrm{O}(\mathrm{N})$ multi-body system dynamics spatial operator algebra (SOA) in reconfigurable spacecraft ground control [4]. This method for large complex machinery systems is becoming increasingly important. Dong Longlei et al established dynamics equation based on Kane dynamics with finite segment modeling method of rigid - flexible coupling multi-body system [5]. Ding Xilun analyzed Lagrange dynamics with Lie algebra to

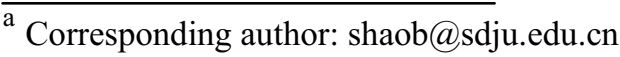

describe two spatial dynamics of a flexible rod robot and compared with ADAMS simulation results [6].

In summary, the rigid system of coupled nonlinear problems appearing in modern machinery and a variety of constraints are required to develop an efficient recursive algorithm. Based on the above methods, this paper dynamics developed the recursive dynamics based on Lie groups and Lie algebras which can be applied to the complex mechanical system. The kinetic equations by recursive methods can achieve efficient real-time dynamic simulation and control. Some examples show the correctness and efficiency of the method.

\section{Recursive dynamics of Lie groups and Lie algebra}

\subsection{Adjoint transformations of Lie groups and Lie algebra}

A Lie group is a group and also it is a differentiable manifold expressed by $\mathrm{G}$. The tangent space of a Lie group's element is called the Lie algebra for that group. The Lie algebra forms a vector space with a bilinear map which is called the Lie bracket [7].

The rotation matrix is an orthogonal matrix in three dimensional Euclidean space and it is an element of the special Orthogonal group $S O(3)$. The homogeneous transformation is the Lie group referred to as the special Euclidean group $S E(3)$. The Lie algebra of $S O(3)$ is $s o(3)$ which consists of the set of skew symmetric matrices on $R^{3 \times 3}$. Similar with it, the Lie algebra of $S E(3)$ is $s e(3)$. The relation between a Lie group $S O(3)$ or $S E(3)$ and the associated Lie algebra $s o(3)$ or $s e(3)$ is the matrix exponential.

A linear mapping on the Lie algebra is an element of the Lie group. This is called the Adjoint map on $S E(3)$ and is denoted by $A d$, where $A d_{G}(h)=G h G^{-1}$, $G \in \operatorname{SE}(3)$ and $h \in \operatorname{se}(3)$. Note that $h$ is the homogeneous matrix. 


$$
A d_{G}(h)=\left[\begin{array}{cc}
R & 0 \\
{[p] R} & R
\end{array}\right]\left[\begin{array}{c}
\omega_{h} \\
v_{h}
\end{array}\right]
$$

The linear mapping on the dual space se(3) is a dual Adjoint operator denoted by $A d^{*}$. For $h^{*}$ :

$$
A d_{G}^{*}\left(h^{*}\right)=\left[\begin{array}{cc}
R^{T} & R^{T}[p]^{T} \\
0 & R^{T}
\end{array}\right]\left[\begin{array}{c}
\mathscr{M} \\
\not
\end{array}\right]
$$

The Lie algebra can also be used as a linear mapping on itself denoted by $a d$. It has the form of $\operatorname{ad}_{g}(h)=[g, h]=g h-h g$, where $g, h \in \operatorname{se}(3)$.

$$
a d_{g}(h)=\left[\begin{array}{cc}
{\left[\omega_{g}\right]} & 0 \\
{\left[v_{g}\right]} & {\left[\omega_{g}\right]}
\end{array}\right]\left[\begin{array}{l}
\omega_{h} \\
v_{h}
\end{array}\right]
$$

Similarly, the dual operator $a d^{*}$ is given by $a d_{g}^{*}\left(h^{*}\right)=\left[g, h^{*}\right]$ and:

$$
a d_{g}^{*}\left(h^{*}\right)=\left[\begin{array}{cc}
{\left[\omega_{g}\right]^{T}} & {\left[v_{g}\right]^{T}} \\
0 & {\left[\omega_{g}\right]^{T}}
\end{array}\right]\left[\begin{array}{c}
\mathscr{M} \\
\boldsymbol{Z}
\end{array}\right]
$$

In multi-body dynamics, the Adjoint operator $\mathrm{Ad}$ maps the spatial velocity screws from one reference frame to body frame and the dual Adjoint operator $A d^{*}$ maps the spatial force screws. The mapping $a d$ and $a d^{*}$ respectively define the standard cross product operation of the 6-vector velocity screws and force screws.

\subsection{Dynamics of tree topology}

\subsubsection{Outward recursion of spatial velocity}

Given $\mathrm{V}_{0}$, outward recursion to compute the velocities in depth first manner:

$$
\begin{aligned}
& \boldsymbol{T}_{P, i}=\boldsymbol{M}_{i} e^{\boldsymbol{S}_{i} q_{i}} \\
& \boldsymbol{V}_{i}=A d_{\boldsymbol{T}_{P, i}^{-1}}\left(\boldsymbol{V}_{P}\right)+\boldsymbol{S}_{i} \dot{q}_{i} \\
& \boldsymbol{a}_{i}=a d_{\boldsymbol{V}_{i}}\left(\boldsymbol{S}_{i} \dot{\mathrm{q}}_{i}\right) \\
& \boldsymbol{b}_{i}=-a d_{\boldsymbol{V}_{i}}^{*}\left(\boldsymbol{J}_{i} \boldsymbol{V}_{i}\right)
\end{aligned}
$$

Where $P$ denotes the index of body $i$ 's parent body.

The principle of inward recursive is based on breadthfirst which begins from the farthest chain endings. Inward recursive calculate all $\mathrm{J}$ and $\mathrm{B}$ of links. It allows any number of external forces applied to the branch chain link.

\subsubsection{Inward recursion of $\hat{\boldsymbol{J}}_{i}$ and $\boldsymbol{B}_{i}$}

Inward recursion to compute the articulated body inertia and articulated body zero force in reversed breadth first manner.

$$
\begin{aligned}
& \hat{\boldsymbol{J}}_{i}=\boldsymbol{J}_{i}+\sum_{j \in C} A d_{\boldsymbol{T}_{i, j}^{-1}}^{*} \hat{\boldsymbol{J}}_{j}^{+} A d_{\boldsymbol{T}_{i, j}^{-1}} \\
& \boldsymbol{B}_{i}=\boldsymbol{b}_{i}+\sum_{j \in C} A d_{\boldsymbol{T}_{i, j}^{-1}}^{*} \boldsymbol{z}_{j}^{+} \\
& \boldsymbol{z}_{i}=\hat{\boldsymbol{J}}_{i} \boldsymbol{a}_{i}+\boldsymbol{B}_{i}+\sum_{j} A d_{T_{i, j}^{-1}}^{*} \boldsymbol{F}_{i, j}^{\prime} \\
& \hat{\boldsymbol{J}}_{i}^{+}=\left[\boldsymbol{I}-\frac{\hat{\boldsymbol{J}}_{i} \boldsymbol{S}_{i} \boldsymbol{S}_{i}^{T}}{\boldsymbol{S}_{i}^{T} \hat{\boldsymbol{J}}_{i} \boldsymbol{S}_{i}}\right] \hat{\boldsymbol{J}}_{i} \\
& \boldsymbol{z}_{i}^{+}=\left[\boldsymbol{z}_{i}+\frac{\hat{\boldsymbol{J}}_{i} \boldsymbol{S}_{i}\left(\tau_{i}-\boldsymbol{S}_{i}^{T} \boldsymbol{z}_{i}\right)}{\boldsymbol{S}_{i}^{T} \hat{\boldsymbol{J}}_{i} \boldsymbol{S}_{\boldsymbol{i}}}\right]
\end{aligned}
$$

Where $C$ denotes the set of child bodies.

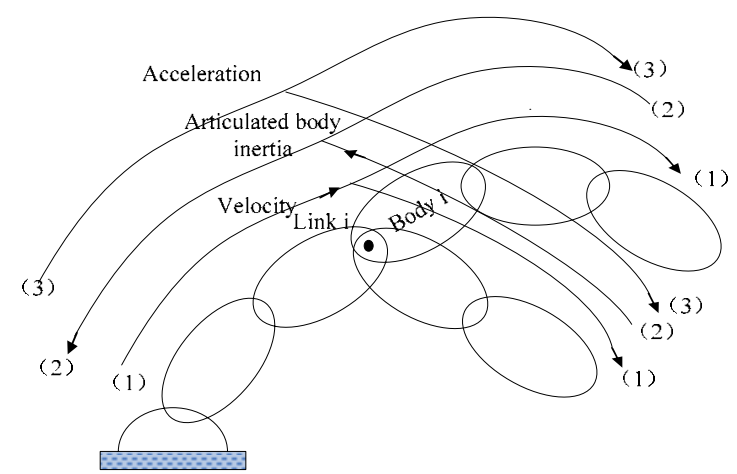

Figure 1. Forward dynamics of tree topology system

\subsubsection{Outward recursion of acceleration}

Given $\dot{V}_{0}$, outward recursion to compute the acceleration in depth first manner:

$$
\begin{aligned}
& \dot{\boldsymbol{V}}_{i}=\hat{\boldsymbol{J}}_{i} A d_{\boldsymbol{T}_{P, i}^{-1}}\left(\dot{\boldsymbol{V}}_{P}\right)+\boldsymbol{S}_{i} \ddot{q}_{i}+\boldsymbol{a}_{i} \\
& \ddot{q}_{i}=\frac{\tau_{i}-\boldsymbol{S}_{i}^{T}\left[\hat{\boldsymbol{J}}_{i} A d_{\boldsymbol{T}_{P, i}^{-1}}\left(\dot{\boldsymbol{V}}_{P}\right)+\boldsymbol{z}_{i}\right]}{\boldsymbol{S}_{i}^{T} \hat{\boldsymbol{J}}_{i} \boldsymbol{S}_{i}}
\end{aligned}
$$

For the complex mechanical systems with closed loop constraints, recursive dynamics method can be constructed by a series of natural orthogonal complement matrix, such that the closed loop system dynamic equations relating the generalized inertia matrix can be clearly shown, and then the generalized inertia matrix recursive decomposition kinetics can be obtained. As for the flexible lightweight mechanical system, a motion can be expressed as relatively flexible synthetic rigid body motion and movement. Using the finite element method to express the deformation of the flexible object, simply expand each symbol to represent rigid body containing modal distortion coordinate with the previous similar recursive dynamics. 


\section{Example}

Each link of the six-bar press are made of steel, having a density of $7.8 \times 10-6(\mathrm{~kg} / \mathrm{mm} 3)$, Young's modulus of $2.07 \times 105(\mathrm{~N} / \mathrm{mm} 2)$, Poisson's ratio of 0.29 . Parameters of each link are shown in Table 1.

Link 4 is the input with the speed of $1.47(\mathrm{rad} / \mathrm{s})$. The simulation curve is shown in figure 2 to figure 5 .

Table 1. Parameters of the six-bar press

\begin{tabular}{|c|c|c|c|c|}
\hline Parameters & Mass(kg) & $\begin{array}{l}\text { Center of } \\
\text { mass } \\
(\mathrm{mm})\end{array}$ & $\begin{array}{l}\text { Inertia (kg } \\
\left.\cdot \mathrm{mm}^{2}\right)\end{array}$ & $\begin{array}{l}\text { Initial } \\
\text { (deg) }\end{array}$ \\
\hline Link 2 & 6.28 & $\begin{array}{l}{[181.1,} \\
452.7, \\
0.0]\end{array}$ & $\begin{array}{l}{\left[5.3 \times 10^{5},\right.} \\
5.3 \times 10^{5}, \\
1039.8]\end{array}$ & 340.9 \\
\hline Link 3 & 9.19 & $\begin{array}{l}{[1103.4,} \\
-143.8, \\
0.0]\end{array}$ & $\begin{array}{l}{\left[1.4 \times 10^{6},\right.} \\
1.4 \times 10^{6}, \\
\left.7.6 \times 10^{4}\right]\end{array}$ & 316.9 \\
\hline Link 4 & 1.69 & $\begin{array}{l}{[1280.0,} \\
251.6, \\
0.0]\end{array}$ & $\begin{array}{l}{\left[1.1 \times 10^{4},\right.} \\
1.0 \times 10^{4}, \\
275.0]\end{array}$ & 0 \\
\hline Link 5 & 5.997 & $\begin{array}{l}{[1209.4,} \\
-1220.9, \\
0.0]\end{array}$ & $\begin{array}{l}{\left[4.6 \times 10^{5},\right.} \\
4.6 \times 10^{5}, \\
992.7]\end{array}$ & 353.2 \\
\hline Slider & 62.41 & $\begin{array}{l}{[1400.0,} \\
-1645.0, \\
0.0]\end{array}$ & $\begin{array}{l}{\left[4.2 \times 10^{5},\right.} \\
4.2 \times 10^{5}, \\
\left.4.2 \times 10^{5}\right]\end{array}$ & \\
\hline
\end{tabular}

As can be seen from the figure, work area of six-link press is longer than ordinary crank press. It can be achieved full capacity in the entire working area, so the six-link press is particularly suitable for deep drawing stretch craft work. Because of the smooth operation of the slider, operating noise is greatly reduced. When the slider reaches the maximum acceleration, the torque of each joint almost simultaneously reached the maximum. Visible dynamics simulation results obtained using the process can be drawn throughout the run all the links and the joints of the forces.

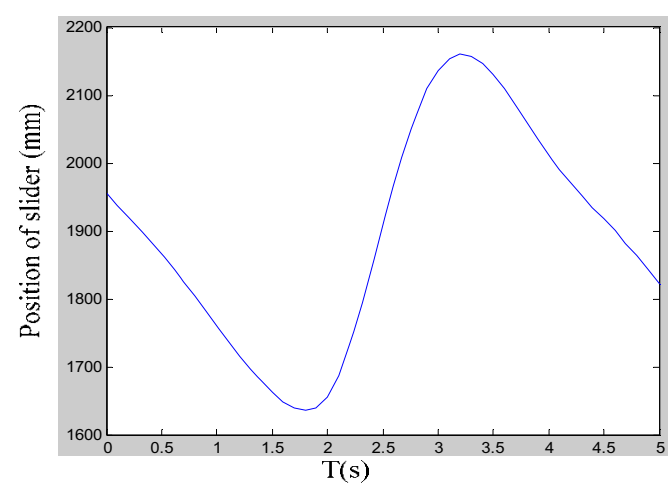

Figure 2. Position of slider

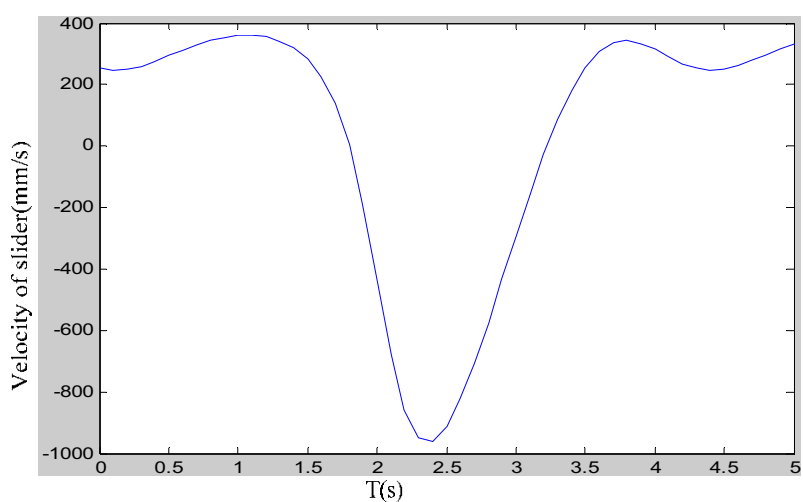

Figure 3. Velocity of slider

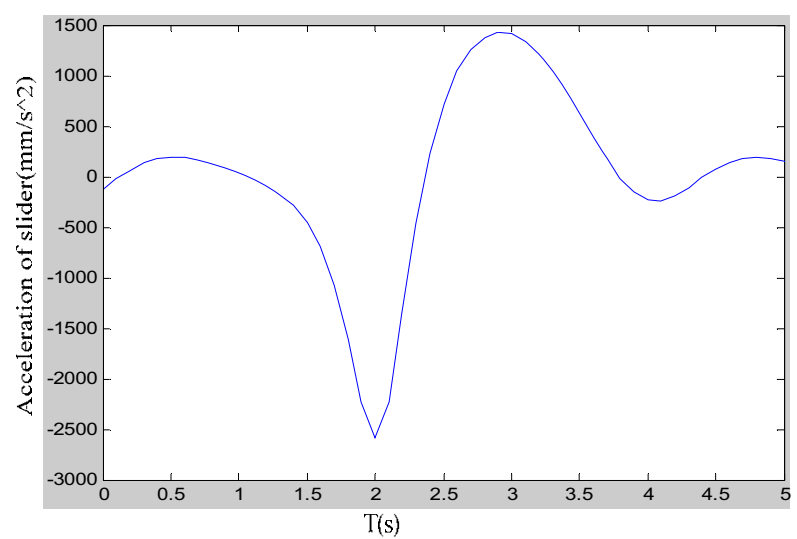

Figur 4. Acceleration of slider

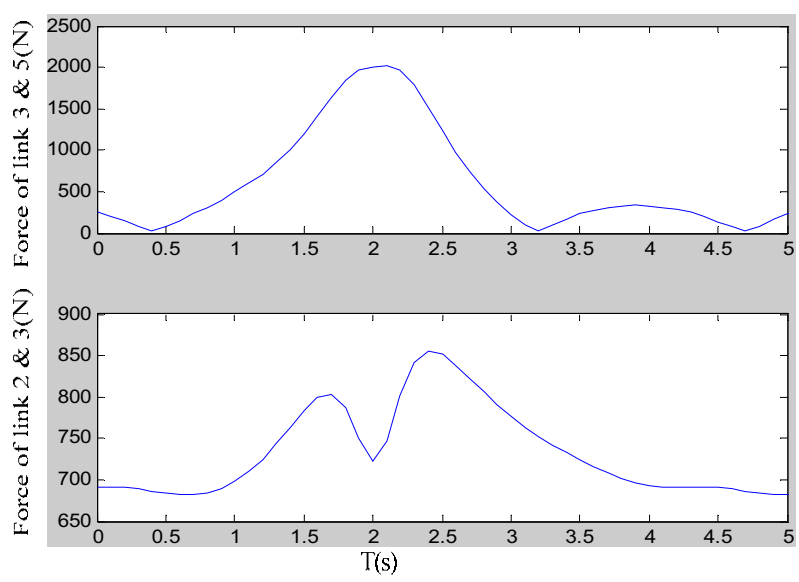

Figure 5. Force of link $2 \& 3$ and link $3 \& 5$ 


\section{Conclusions}

In this paper, Li Lie algebra theory is used to describe complex mechanical system. This efficient recursive dynamics is not only suitable for the chain, tree topology recursive mechanical system, and can be further extended to the closed-loop and flexible system. Its complexity is $\mathrm{O}(\mathrm{N})$. This concise, clear and efficient algorithm is very important for real-time dynamics. Virtual simulation positive dynamics achieved can save huge laboratory fees. Examples of use of the above kinetic method are validated.

\section{Acknowledgment}

This research is partially supported by Young Teacher Training Project Funded Plan of Colleges of Shanghai (project number:ZZSDJ12005) and Scientific Research Project Start-up Fund of Shanghai Dianji University (project number: 11c404).

\section{References}

1. Liu ZY, Hong JZ. Current Situation and Prospect of system dynamics of flexible multi-body. Computational Mechanics, 25, 411 (2008)

2. Hwang Y L. Recursive Newton-Euler formulation for flexible dynamic manufacturing analysis of open loop robotic systems. International Journal of Advanced Manufacturing Technology, 29, 598 (2006)

3. Feather stone R. Efficient factorization of the joint space inertia matrix for branched kinematical trees. International Journal of Robotics Research, 24, 487 (2005)

4. Mukherjee R M, Jain A. Parallelization of the spatial operator algebra for dynamics of multi-body systems. 8th .World Congress on Computational Mechanics .Venice, Italy, 269 (2008)

5. Dong LL, Yan GR, Yu JJ. Finite Segment Modeling of the flexible member with a center of rotation of the rigid body. Xi'an Jiaotong University, 35, 977 (2001)

6. Ding HL, Liu Y. Lie group with Lie algebra analysis robot has a flexible deformable rod space dynamics. Mechanical Engineering, 43, 184 (2007)

7. Park F C, Bobrow J E, Ploen S R. A lie group formulation of robot dynamics. International Journal of Robotics Research, 14, 609 (1995)

8. Sohl G A, Bobrow J E .A recursive multibody dynamics and sensitivity algorithm for branched kinematical chains. Journal of Dynamic Systems, Measurement , and Control, 123, 391 (2001) 